\title{
Interacting relativistic quantum dynamics for multi-time wave functions
}

\author{
Matthias Lienert ${ }^{1}$ a \\ ${ }^{1}$ Mathematical Institute, Ludwig-Maximilians-University, Theresienstr. 39, 80333 Munich, Germany
}

\begin{abstract}
In this paper, we report on recent progress about a rigorous and manifestly covariant interacting model for two Dirac particles in $1+1$ dimensions $[9,10]$. It is formulated using the multi-time formalism of Dirac, Tomonaga and Schwinger. The mechanism of interaction is a relativistic generalization of contact interactions, and it is achieved going beyond the usual functional-analytic Hamiltonian method.
\end{abstract}

\section{Introduction}

The multi-time formalism has the virtue to provide a manifestly covariant representation of the quantum state in the Schrödinger picture. The basic idea, suggested early on by the Nobel laureates Dirac [1], Tomonaga [2] and Schwinger [3] is to regard the usual single-time wave function of the $N$-particle Schrödinger equation,

$$
\varphi: \mathbb{R}^{3 N} \times \mathbb{R} \longrightarrow \mathcal{S}, \quad\left(\mathbf{x}_{1}, \ldots, \mathbf{x}_{N}, t\right) \longmapsto \varphi\left(\mathbf{x}_{1}, \ldots, \mathbf{x}_{N}, t\right),
$$

where $\mathcal{S}$ is a spin space, as the special case of a more general object,

$$
\psi: \Omega \subset \mathbb{R}^{4 N} \longrightarrow \mathcal{S}, \quad\left(x_{1}, \ldots, x_{N}\right) \longmapsto \psi\left(x_{1}, \ldots, x_{N}\right),
$$

the multi-time wave function $\left(x_{k}=\left(t_{k}, \mathbf{x}_{k}\right)\right)$, namely the special case of equal times, i.e.

$$
\varphi\left(\mathbf{x}_{1}, \ldots, \mathbf{x}_{N}, t\right)=\psi\left(t, \mathbf{x}_{1}, \ldots, t, \mathbf{x}_{N}\right) .
$$

In (2), $\Omega$ denotes a suitable domain. Most naturally, it is given by the set of space-like configurations [4-6], as a collection of $N$ points which are space-like related is the most natural relativistic generalization of " $N$ space-points at a time".

While the transformation properties of $\varphi$ under Lorentz transformations $\Lambda \in \mathcal{L}_{+}^{\uparrow}$ cannot easily be defined, the ones of $\psi$ are straightforwardly given by:

$$
\psi^{\prime}\left(x_{1}, \ldots, x_{N}\right)=S[\Lambda] \otimes \cdots \otimes S[\Lambda] \psi\left(\Lambda^{-1} x_{1}, \ldots, \Lambda^{-1} x_{N}\right),
$$

where the matrices $S[\Lambda]$ form a spinor representation of the Lorentz group.

\footnotetext{
a e-mail: lienert@math.lmu.de
} 
The multi-time wave function is assumed to attain physical meaning by a generalized Born rule, i.e. the postulate that a suitable quadratic expression in $\psi\left(x_{1}, \ldots, x_{N}\right)$ yields the probability density to find particle 1 at $x_{1} \in \Sigma$, particle 2 at $x_{2} \in \Sigma$ and so on, for any space-like hypersurface $\Sigma[2,4]$.

As evolution equations, one usually considers $N$ simultaneous Schrödinger equations of Hamiltonian form, each of which expresses the time evolution in one of the $t_{k}$ 's:

$$
i \frac{\partial}{\partial t_{k}} \psi=H_{k} \psi, \quad k=1, \ldots, N,
$$

where the $H_{k}$ are differential operators on an appropriate function space. The familiar Schrödinger equation for $\varphi$ naturally follows from (5) using (3) and the chain rule.

However, in contrast to the usual Schrödinger equation, (5) is not always consistent but possesses a joint solution $\psi$ for every initial $\psi_{0}=\psi\left(t_{1}=0, \ldots, t_{N}=0\right)$ if and only if [7]:

$$
\left[i \partial_{t_{j}}-H_{j}, i \partial_{t_{k}}-H_{k}\right]=0 \forall j, k .
$$

In fact, this consistency condition is fairly restrictive. It was for example recently shown in [7] that if

$$
H_{k}=H_{k}^{\text {Dirac }}+V_{k}\left(x_{1}, \ldots, x_{N}\right),
$$

where $H_{k}^{\text {Dirac }}$ is the free Dirac Hamiltonian acting on the coordinates and spin index of the $k$-th particle and $V_{k}\left(x_{1}, \ldots, x_{N}\right)$ is a potential acting on the spin index of the $k$-th particle, then the multi-time evolution equations are gauge-equivalent to the case of purely external potentials $V_{k} \equiv V_{k}\left(x_{k}\right)$.

Therefore, the question of alternative mechanisms of interaction arises. Instead of going to quantum field theory (QFT) and trying to face the ultraviolet divergence problem there (see $[5,8]$ for multi-time formulations of QFTs), we explore a different possibility to construct a rigorous model here: relativistic contact interactions.

The main idea is that boundary conditions may lead to interaction effects even though the formal operators $H_{k}$ in (5) are the free ones. This clearly avoids the use of potentials and therefore the beforementioned no-go theorem. However, remains a non-trivial task to implement the idea, as Hamiltonian methods are not suitable to treat multi-time equations on a bounded domain $\Omega$ such as the space-like configurations. We shall present an alternative way here. The main virtues of the considered model are its rigorous, interacting and manifestly covariant character as well as its relative simplicity when compared with other rigorous interacting models. (It is constructed so as to be explicitly solvable.)

\section{Definition of the model}

Consider two mass-less spin- $\frac{1}{2}$ particles in $1+1$ dimensions. As mentioned before, the natural domain is given by the set of space-like configurations, i.e.

$$
\Omega:=\left\{\left(t_{1}, z_{1}, t_{2}, z_{2}\right) \in \mathbb{R}^{2} \times \mathbb{R}^{2}:\left(t_{1}-t_{2}\right)^{2}-\left(z_{1}-z_{2}\right)^{2}<0\right\} .
$$

The multi-time wave function is a map

$$
\psi: \Omega \longrightarrow \mathbb{C}^{2} \otimes \mathbb{C}^{2}
$$

and we denote its spin components by $\psi_{i}\left(t_{1}, z_{1}, t_{2}, z_{2}\right), i=1,2,3,4$.

Note that $\Omega$ is the disjoint union of the two open sets $\Omega_{1}$, defined by $z_{1}<z_{2}$, and $\Omega_{2}$, defined by $z_{1}>z_{2}$. Because of this feature, one may choose to consider each $\Omega_{i}$ separately, which we do in the 
following, focusing on $\Omega_{1}$.

As evolution equations, we prescribe the free mass-less Dirac equations for each particle, i.e.

$$
i \gamma_{k}^{\mu} \partial_{k, \mu} \psi\left(x_{1}, x_{2}\right)=0, k=1,2
$$

in covariant notation, or

$$
\begin{aligned}
i \frac{\partial}{\partial t_{1}} \psi\left(t_{1}, z_{1}, t_{2}, z_{2}\right) & =-i \sigma_{3} \otimes 1_{2} \frac{\partial}{\partial z_{1}} \psi\left(t_{1}, z_{1}, t_{2}, z_{2}\right), \\
i \frac{\partial}{\partial t_{2}} \psi\left(t_{1}, z_{1}, t_{2}, z_{2}\right) & =-i 1_{2} \otimes \sigma_{3} \frac{\partial}{\partial z_{2}} \psi\left(t_{1}, z_{1}, t_{2}, z_{2}\right) .
\end{aligned}
$$

when solved for the time derivatives, as in eq. (5). Here, $1_{2}$ stands for the $2 \times 2$ unit matrix,

$$
\gamma^{0}=\sigma_{1}=\left(\begin{array}{ll}
0 & 1 \\
1 & 0
\end{array}\right), \quad \gamma^{1}=\sigma_{1} \sigma_{3}=\left(\begin{array}{cc}
0 & -1 \\
1 & 0
\end{array}\right),
$$

and $\sigma_{i}, i=1,2,3$ denote the Pauli matrices.

Initial data are given on an equal-time hyperplane, e.g. for $t_{1}=t_{2}=0$ :

$$
\psi_{i}\left(0, z_{1}, 0, z_{2}\right)=g_{i}\left(z_{1}, z_{2}\right), z_{1}<z_{2}\left(\text { on } \Omega_{1}\right),
$$

where $g_{i}$ are given $C^{1}$-functions.

Finally, boundary conditions are prescribed (as limits) on the set of coincidence points

$$
C:=\left\{\left(t_{1}, z_{1}, t_{2}, z_{2}\right) \in \mathbb{R}^{2} \times \mathbb{R}^{2}: t_{1}=t_{2}, z_{1}=z_{2}\right\} .
$$

In $[6,9]$ it was shown that a both physically preferred and mathematically admissible class of boundary conditions is given by (using the basis in spin space corresponding to (11), (12)):

$$
\lim _{\varepsilon \rightarrow 0} \psi_{2}(t, z-\varepsilon, t, z+\varepsilon)=e^{-i \theta} \lim _{\varepsilon \rightarrow 0} \psi_{3}(t, z-\varepsilon, t, z+\varepsilon), \quad t, z \in \mathbb{R} .
$$

for any $\theta \in[0,2 \pi)$. The limiting process is well-defined, as we aim at $C^{1}$-solutions (in both space and time). We shall explain the special features of this class of boundary conditions in the following.

\section{Results}

\subsection{Probability conservation}

In order for a quantum-mechanical equation to have statistical meaning, probability conservation has to be ensured. The system (10) possesses the conserved tensor current

$$
j^{\mu \nu}\left(x_{1}, x_{2}\right)=\bar{\psi}\left(x_{1}, x_{2}\right) \gamma_{1}^{\mu} \gamma_{2}^{v} \psi\left(x_{1}, x_{2}\right)
$$

where $\bar{\psi}:=\psi^{\dagger} \gamma_{1}^{0} \gamma_{2}^{0}$. "Conserved" means $\partial_{1, \mu} j^{\mu v}=\partial_{2, \nu} j^{\mu v}=0$. Furthermore, $j^{\mu v}$ is positive-definite as $j^{00}=\psi^{\dagger} \psi \geq 0$. Therefore, we can express probability conservation on a general space-like hypersurface $\Sigma$ (and in the domain $\Omega_{1}$ ) as [9]:

$$
\int_{(\Sigma \times \Sigma) \cap \Omega_{1}} d \sigma_{1, \mu} d \sigma_{2, v} j^{\mu v}\left(x_{1}, x_{2}\right)=1, \quad \text { independently of } \Sigma \text {. }
$$

By treating $d \sigma_{1, \mu} d \sigma_{2, v} j^{\mu \nu}\left(x_{1}, x_{2}\right)$ as an $2 d$-form $(d=1$ here), constructing a closed surface including $\left(\Sigma_{i} \times \Sigma_{i}\right) \cap \Omega_{1}$ for two different space-like hypersurfaces $\Sigma_{i}, i=1,2$ and using Stokes' theorem in its differential geometric form, one can show [9]:

Theorem 3.1 Probability conservation on $\Omega_{1}$ in the sense of (17) is ensured by the boundary conditions (15). 


\subsection{Lorentz invariance}

The main goal of the multi-time formulation is manifest covariance. Apart from the boundary conditions, the Lorentz invariance of the model is already clear from its definition. In order to check the covariance of the boundary conditions, we explicitly determine the matrices $S[\Lambda]$ in (4).

According to the representation theory of Dirac spinors, we have:

$$
S[\Lambda]=\exp \left(\frac{\beta}{4}\left[\gamma^{0}, \gamma^{1}\right]\right), \beta \in \mathbb{R} .
$$

Using the representation (12), we explicitly obtain:

$$
S[\Lambda]=\left(\begin{array}{cc}
\cosh \beta+\sinh \beta & 0 \\
0 & \cosh \beta-\sinh \beta
\end{array}\right)
$$

and it follows:

$$
S[\Lambda] \otimes S[\Lambda]=\left(\begin{array}{llll}
(\cosh \beta+\sinh \beta)^{2} & & & \\
& 1 & & \\
& & 1 & \\
& & & (\cosh \beta-\sinh \beta)^{2}
\end{array}\right) .
$$

Therefore, under a Lorentz transformation $\Lambda$, the components $\psi_{2}, \psi_{3}$ transform as:

$$
\psi_{i}\left(x_{1}, x_{2}\right) \stackrel{\Lambda}{\longmapsto} \psi_{i}\left(\Lambda^{-1} x_{1}, \Lambda^{-1} x_{2}\right) \text { for } i=2,3 .
$$

Because in addition the boundary conditions are prescribed on the Lorentz invariant set $C$, we obtain:

Theorem 3.2 The model, as defined by (8), (10), (13) and (15), is Lorentz invariant.

\subsection{Existence and uniqueness of solutions}

The previous two subsections have established some of the most important physical properties of the model. Of course, also mathematical sense has to be given to it. This requires to establish the existence and uniqueness of solutions of the initial boundary value problem (11), (13) and (15).

In order to understand the meaning of the multi-time evolution equations (11) in more detail, we write them out in matrix-vector form:

$$
\begin{aligned}
i \frac{\partial}{\partial t_{1}}\left(\begin{array}{l}
\psi_{1} \\
\psi_{2} \\
\psi_{3} \\
\psi_{4}
\end{array}\right) & =-i\left(\begin{array}{cccc}
1 & & & \\
& 1 & & \\
& & -1 & \\
& & & -1
\end{array}\right) \frac{\partial}{\partial z_{1}}\left(\begin{array}{l}
\psi_{1} \\
\psi_{2} \\
\psi_{3} \\
\psi_{4}
\end{array}\right), \\
i \frac{\partial}{\partial t_{2}}\left(\begin{array}{l}
\psi_{1} \\
\psi_{2} \\
\psi_{3} \\
\psi_{4}
\end{array}\right) & =-i\left(\begin{array}{llll}
1 & & & \\
& -1 & & \\
& & 1 & \\
& & & -1
\end{array}\right) \frac{\partial}{\partial z_{2}}\left(\begin{array}{l}
\psi_{1} \\
\psi_{2} \\
\psi_{3} \\
\psi_{4}
\end{array}\right) .
\end{aligned}
$$

For example, for $\psi_{1}$ we have:

$$
\left(\frac{\partial}{\partial t_{1}}+\frac{\partial}{\partial z_{1}}\right) \psi_{1}=0, \quad\left(\frac{\partial}{\partial t_{2}}+\frac{\partial}{\partial z_{2}}\right) \psi_{1}=0 \Rightarrow \psi_{1}\left(t_{1}, z_{1}, t_{2}, z_{2}\right)=f_{1}\left(z_{1}-t_{1}, z_{2}-t_{2}\right)
$$


where $f_{1}$ is a $C^{1}$-function.

We see that each component of $\psi$ is constant along certain two-dimensional surfaces in fourdimensional space, e.g. defined by $z_{1}-t_{1}=c_{1}, z_{2}-t_{2}=c_{2}$ for $\psi_{1}$ and constants $c_{1}, c_{2} \in \mathbb{R}$. In analogy to the method of characteristics in PDE theory, we call these surfaces the multi-time characteristics of $\psi$. They allows for a powerful method to study the existence and uniqueness theory of our model [9]. To see whether $\psi_{i}$ is uniquely determined at a point $p \in \Omega_{1}$, one considers the multi-time characteristic which includes $p$. Then one calculates whether it intersects either the boundary set $C$ or the initial data surface $t_{1}=t_{2}=0$ in a single point $q$. If for all $i$ and all $p$ there exists a path connecting $p$ with the respective $q$ while staying in the multi-time characteristic intersected with the domain $\Omega_{1}$, then there exists a unique solution of the initial boundary value problem. At the boundary, it has to be made clear, in addition, that for every $t$ exactly one side of (15) is already uniquely determined by initial conditions.

By this method, it is even possible to determine the explicit solution [6, lemma 3.6.1]:

Theorem 3.3 The unique solution of the initial boundary value problem (11), (13) and (15) on $\Omega_{1}$, where the initial data are supposed to satisfy the boundary conditions, is given by:

$$
\begin{aligned}
& \psi_{1}\left(t_{1}, z_{1}, t_{2}, z_{2}\right)=g_{1}\left(z_{1}-t_{1}, z_{2}-t_{2}\right), \\
& \psi_{2}\left(t_{1}, z_{1}, t_{2}, z_{2}\right)= \begin{cases}g_{2}\left(z_{1}-t_{1}, z_{2}+t_{2}\right) & \text { for } z_{1}-t_{1}<z_{2}+t_{2} \\
e^{-i \theta} g_{3}\left(z_{2}+t_{2}, z_{1}-t_{1}\right) & \text { for } z_{1}-t_{1} \geq z_{2}+t_{2}\end{cases} \\
& \psi_{3}\left(t_{1}, z_{1}, t_{2}, z_{2}\right)= \begin{cases}g_{3}\left(z_{1}+t_{1}, z_{2}-t_{2}\right) & \text { for } z_{1}+t_{1}<z_{2}-t_{2} \\
e^{i \theta} g_{2}\left(z_{2}-t_{2}, z_{1}+t_{1}\right) & \text { for } z_{1}+t_{1} \geq z_{2}-t_{2}\end{cases} \\
& \psi_{4}\left(t_{1}, z_{1}, t_{2}, z_{2}\right)=g_{4}\left(z_{1}+t_{1}, z_{2}+t_{2}\right) .
\end{aligned}
$$

\subsection{Interaction}

The main point about the model is to achieve a mechanism of interaction by boundary conditions. Of course, the question of how to decide whether interaction is present arises. In non-relativistic quantum mechanics, one simply defines interactions by the presence of an interaction potential. Of course, such a definition is limited to a particular mathematical framework and is not general enough here. Instead, we try to capture the essence of interaction as follows: A model is said to be interacting if it generates entanglement, i.e. if there are initial product wave functions $\psi\left(0, z_{1}, 0, z_{2}\right)=\phi\left(z_{1}\right) \otimes \chi\left(z_{2}\right)$ which cannot be written as product wave functions for later times $t_{1}, t_{2}$.

With the aid of the explicit solution formula, this criterion is easy to check. Using

$$
\begin{aligned}
& g_{1}\left(z_{1}, z_{2}\right)=\phi_{1}\left(z_{1}\right) \chi_{1}\left(z_{2}\right), \quad g_{2}\left(z_{1}, z_{2}\right)=\phi_{1}\left(z_{1}\right) \chi_{2}\left(z_{2}\right) \\
& g_{3}\left(z_{1}, z_{2}\right)=\phi_{2}\left(z_{1}\right) \chi_{1}\left(z_{2}\right), \quad g_{4}\left(z_{1}, z_{2}\right)=\phi_{2}\left(z_{1}\right) \chi_{2}\left(z_{2}\right)
\end{aligned}
$$

in (24), one indeed finds, as a consequence of the case differentiation for $\psi_{2}, \psi_{3}$ :

Theorem 3.4 The model defined by (11), (13) and (15) on $\Omega_{1}$ is interacting.

By studying the behavior of localized wave packets in more detail (compare [9]), one can see that the interaction corresponds to a scattering process with range zero, where the two particle bounce off each other when they meet. In addition, the scattering is associated with a spin flip and relative phase shift of the components $\psi_{2}, \psi_{3}$ corresponding to $|\uparrow \downarrow\rangle \leftrightarrow e^{-i \theta}|\downarrow \uparrow\rangle$. 


\section{Summary and outlook}

Indeed, a rigorous interacting relativistic model could be constructed, conforming to the strictest requirements of relativistic invariance, as embodied by the multi-time formalism. Its relative simplicity when compared with other models of this kind makes it particularly attractive as a pedagogical example. It is, furthermore, a counterexample to the claim in the "folklore" of relativistic quantum mechanics that the creation and annihilation of particles were necessary form a purely mathematical point of view to achieve relativistic interactions.

The developed methods, such as the formulation of probability conservation for $N$ particles on space-like hypersurfaces as well as the generalized method of characteristics, may be of interest for both scientists working in relativistic quantum mechanics as well as mathematical physics.

Besides this, one may ask whether or how the model can be generalized to more realistic situations. As shown in [10], the extension to $N \geq 3$ particles is straightforward. The formulation of a similar model for higher dimensions, however, does not seem feasible, as the set of coincidence points then is too low-dimensional to have impact on the time evolution [9].

It will be an interesting future task to develop further generalizations with respect to the massive case as well as to a variable particle number. The assumption of zero masses has so far been a technical one whose main advantage is to render the model explicitly solvable. However, besides the strategy for the existence proof, nothing essential is expected to change for non-zero masses, since the boundary conditions are extracted from the tensor current $j^{\mu \nu}$ which stays the same in the massive case. With regard to the variable particle number case, the idea would be to choose the particle-position representation of QFT (see [5]) such that $\psi=\left(\psi^{(0)}, \psi^{(1)}, \psi^{(2)}, \ldots\right)$ where $\psi^{(n)}$ is an $n$-particle wave function. When compared to the $N$-particle case, additional possibilities for the boundary conditions appear, namely by relating $\psi^{(n)}$ with $\psi^{(m)}$ for $n \neq m$. This corresponds to the recently introduced idea of "interior boundary conditions" which was used in $[11,12]$ to avoid ultraviolet divergencies for a simple non-relativistic QFT. Studying the variable particle number case for our model may help to extend this idea to a relativistic setting.

\section{References}

[1] P. A. M. Dirac. Proc. R. Soc. Lond. A, 136:453-464, 1932.

[2] S. Tomonaga. In J. Schwinger, editor, Selected Papers on Quantum Electrodynamics, pages 156168. Dover, 1958.

[3] J. Schwinger. Phys. Rev., 74(2162):1439-1461, 1948.

[4] F. Bloch. Phys. Z. d. Sowjetunion, 5:301-315, 1934.

[5] S. Petrat and R. Tumulka. Ann. Phys., 345:17-54, 2014.

[6] M. Lienert. PhD thesis, 2015. Mathematical Institute, Ludwig-Maximilians-University, Munich, Germany. https://edoc.ub.uni-muenchen.de/18705/.

[7] S. Petrat and R. Tumulka. J. Math. Phys., 55(032302), 2014.

[8] S. Petrat and R. Tumulka. J. Phys. A, 47(11):112001, 2014.

[9] M. Lienert. J. Math. Phys., 56(4):042301, 2015.

[10] M. Lienert and L. Nickel. J. Phys. A, 48(32):325301, 2015. arXiv:1502.00917.

[11] S. Teufel and R. Tumulka. Preprint: arXiv:1505.04847 (2015)

[12] S. Teufel and R. Tumulka. To appear in F. Finster, J. Kleiner, C. Röken, and J. Tolksdorf (editors), Quantum Mathematical Physics - A Bridge between Mathematics and Physics, Basel: Birkhäuser (2015). Preprint: arXiv:1506.00497. 